Recepción: 27/10/2015

Aceptación: 27/07/2016

Publicación: 24/08/2016

\title{
DESARROLLO DE HABILIDADES MEDIANTE EL APRENDIZAJE AUTÓNOMO
}

\section{DEVELOPMENT OF SKILLS THROUGH AUTONOMOUS LEARNING}

Francisco Javier Cárcel Carrasco ${ }^{1}$

1. Doctor Ingeniero Industrial. Universitat Politècnica de València, Camino de Vera S/N, 46022, Valencia, España.

E-mail: fracarc1@csa.upv.es

\section{Citación sugerida:}

Cárcel Carrasco, F.J. (2016). Desarrollo de habilidades mediante el aprendizaje autónomo. $3 C$ Empresa, investigación y pensamiento crítico, 5(3), 54-62. DOI:

$<$ http://dx.doi.org/10.17993/3cemp.2016.050327.63-85/>. 


\section{RESUMEN}

La educación basada en el desarrollo de competencias es la educación que busca desarrollar integralmente habilidades alcanzables mediante estrategias de aprendizaje que produzcan en el estudiante un aprendizaje autónomo. Desarrollar habilidades para el aprendizaje autónomo motiva al alumno para emprender estudios posteriores con mayor autonomía. Fomentando habilidades de auto-aprendizaje eficiente, el alumno adquiere capacidades para la adaptación y actualización de nuevos conocimientos y avances técnicos, adoptando una aptitud creativa. En contraste frente a la lección magistral, el trabajo autónomo de los estudiantes permite que sigan su propio ritmo adaptando el aprendizaje a sus características particulares, desarrollando habilidades para tomar decisiones, innovar y resolver problemas.

\section{ABSTRACT}

The development of competence-based education is education which aims to develop fully attainable skills by learning strategies that produce in the student autonomous learning. Develop skills for autonomous learning encourages students to undertake further studies with greater autonomy. Promoting efficient self-learning skills, the student acquires capacities for adaptation and updating of new knowledge and technical advances, by adopting a creative aptitude. In contrast to the Masterclass, the independent work of students allows you to follow your own pace learning adapted to their particular characteristics, to develop skills to make decisions, innovate and solve problems.

\section{PALABRAS CLAVE}

Aprendizaje autónomo; autoaprendizaje; formación

\section{KEY WORDS}

Autonomous learning; self-learning; training 


\section{INTRODUCCIÓN}

El aprendizaje autónomo es un proceso donde la propia persona autorregula su aprendizaje y toma conciencia de sus propios procesos cognitivos y socio-afectivos. El esfuerzo pedagógico en este caso está orientado hacia la formación de sujetos centrados en resolver aspectos concretos de su propio aprendizaje, y no sólo en resolver una tarea determinada, es decir, orientar al estudiante a que se cuestione, revise, planifique, controle y evalúe su propia acción de aprendizaje (Martínez, 2004). El proceso de enseñanza tiene como objetivo desarrollar conductas potenciando niveles altos de comprensión y de control del aprendizaje por parte de los alumnos (Martí, 2000).

En las nuevas tendencias pedagógicas el estudiante debe tener un rol en el que debe ser el elemento principal de su aprendizaje a través de controlar y tomar sus propias decisiones del proceso (Pérez de Cabrera, 2013). En el enfoque por competencias, el estudiante es "el fin y el centro del aprendizaje (Vásquez, 2001). Los roles del estudiante autónomo exigen de él un alto grado de involucramiento en las actividades pedagógicas; tal involucramiento no se da al azar sino más bien se basa en razonamientos que le preparan para su futuro profesional (Pérez de Cabrera, 2013).

En el presente trabajo se presenta las características básicas para un sistema de aprendizaje autónomo como estrategia para la enseñanza.

\section{LOS CONCEPTOS DEL APRENDIZAJE AUTÓNOMO}

El aprendizaje autónomo requiere de un enlace entre las variables cognitivas y las afectivo/motivacionales, que se deben tener presentes en el momento de diseñar y aplicar estrategias de aprendizaje (Sanz, 1998).

Lo anterior implica promover que el alumno reflexione sobre su conocimiento, y también sobre sus procesos motivacionales, es decir, tiene que ser consciente de qué lo motiva a aprender, ante los retos que le representa una tarea propuesta.

Los sujetos con un buen nivel de autorregulación deben ser capaces de:

- Mejorar sus habilidades de aprendizaje a través del uso de estrategias motivacionales.

- Seleccionar y crear ambientes adecuados para el aprendizaje.

- Seleccionar la forma y cantidad de instrucción que necesitan aprender.

- Tomar conciencia de la calidad de su aprendizaje en función de sus propias limitaciones y logros. 
Los alumnos deben aprender a planificar, monitorear y valorar de manera consciente las actitudes y limitaciones con respecto a las demandas cognoscitivas de una tarea específica. Por lo que es necesario (Crispín, 2011):

1. Planear: establecer metas y actividades que posibiliten el cumplimiento de la tarea.

2. Monitorear: incluye la comprensión de cómo se está realizando la tarea y la redirección de las estrategias que se utilizan, si fuese necesario.

3. Valorar: es la comprensión de la eficacia y la eficiencia con la que se desarrolla la actividad de aprendizaje. Permite valorar qué tanto el esfuerzo realizado se corresponde con los resultados obtenidos.

El proceso de autorregulación es un proceso complejo, multicausal y multidimensional. Los constructivistas, en un esfuerzo por comprenderlo, consideran los siguientes procesos:

a. Procesos propios de las tareas:

Definición de metas para orientar al sujeto en la cantidad y calidad del esfuerzo necesario.

Estructura de las tareas para identificar y precisar lo que debe ser aprendido.

b. Procesos propios de los sujetos:

Autoconocimiento, definido como la comprensión que poseen los sujetos de sus propias capacidades.

Autoeficacia, o creencia en que las conductas correctas, mediante un esfuerzo razonable, pueden conducir al éxito de la tarea.

c. Procesos propios de las estrategias de aprendizaje:

Desarrollar el uso de diferentes estrategias, que dependerán de las tareas y metas específicas.

Es necesario tener claridad en las metas, de manera que esto oriente a la persona en la cantidad y calidad de esfuerzo necesario para lograrlas. El funcionamiento efectivo del aprendizaje no se alcanza sólo consiguiendo el conocimiento específico sobre un dominio, sino que lo más relevante es el conocimiento metacognitivo acerca del mismo, aspecto que se ve favorecido si existe claridad en las metas de aprendizaje (Martínez, 2004).

\section{PLANIFICACIÓN DEL APRENDIZAJE AUTÓNOMO}

Cuando el profesor diseña los objetivos de aprendizaje debe contextualizarlos, es decir, pensar quiénes son los estudiantes, qué deben aprender y por qué. Necesita ubicar la materia dentro del plan de estudios y aclarar qué conocimientos previos tienen los estudiantes y de qué manera lo que aprenderán contribuirá a la formación del egresado. 
Lo recomendable es que antes de diseñar las actividades de aprendizaje, el profesor diseñe la evaluación para darse cuenta como se orientan el logro de objetivos y la coherencia con la evaluación. Creando una alineación con:

- La creación de un ambiente propicio para el aprendizaje.

- Conducir los procesos de enseñanza y aprendizaje.

- Evaluación.

Para que los alumnos aprendan a utilizar las estrategias de aprendizaje adecuadas según el caso, el profesor debe seguir un proceso para mejorar la enseñanza del pensamiento que pasa por tres etapas (Monereo, 2001):

a. Presentar y modelar la estrategia

Las estrategias de aprendizaje pueden aprenderse a través del profesor, para lo cual el docente necesita hacer una reflexión acerca de las propias estrategias que emplea para aprender determinados contenidos, y hacer explícitas las estrategias que está utilizando.

b. Práctica guiada

El siguiente paso, después de presentar y modelar la estrategia debe haber una práctica guiada. Por ejemplo, para encontrar las ideas principales de un texto el profesor explica cómo lo hace, luego da algunas pautas para que los estudiantes lo ejerciten, en forma de guías con ciertas preguntas clave. Más tarde se da oportunidad a los estudiantes para que practiquen la estrategia en distintos contextos. Se debe procurar que reflexionen sobre su propia experiencia de manera que, poco a poco, vayan sabiendo cuándo, cómo y por qué usar determinada estrategia para realizar determinadas tareas o cumplir ciertas metas (figura 1).

\section{Más dependencia}

ALUMNO

Menos dependencia

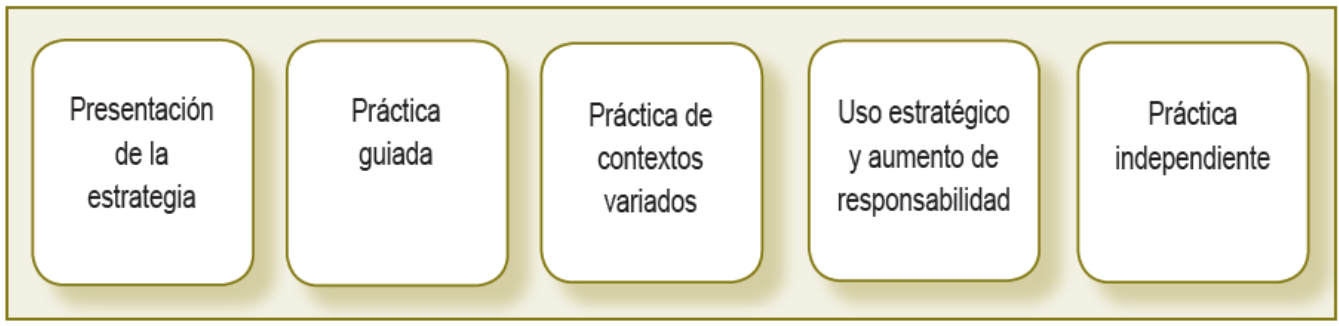

Más control de la actividad

\section{PROFESOR}

Menos control de

la actividad

Figura 1. Enseñanza y aprendizaje de estrategias de aprendizaje.

Fuente: Crispín, 2011. 
Los estudiantes deben aprender a tomar conciencia de las estrategias que utilizan al aprender, al resolver un problema o al realizar una tarea de aprendizaje. El estudiante toma conciencia de sus propios procesos y aprender otras estrategias para resolver un mismo problema.

La participación guiada, la asistencia y retroalimentación continua por parte del profesor paulatinamente decreciente, favorece que el estudiante mejore sus propias estrategias y tenga un mayor control sobre su propio aprendizaje.

\section{EL CONTRATO DE APRENDIZAJE}

En todo proceso de aprendizaje existe un discurso entre profesor y alumno resultado del conjunto de códigos y pactos implícitos y explícitos que regulan los comportamientos.

Existe un contrato de aprendizaje cuando alumno/a, y profesor/a de forma explícita intercambian sus opiniones, comentan sus necesidades, comparten proyectos y deciden en colaboración la forma de llevar a cabo el proceso de enseñanza y lo reflejan oralmente o por escrito. Se podrían especificar los principios fundamentales como (García \& Fortea, 2006):

(1) Consentimiento mutuo. Ante una oferta se produce una respuesta con una aceptación o demanda y si consienten se inicia el proceso.

(2) Aceptación positiva del alumno, en tanto que es quien mejor se conoce y quien en definitiva realiza la actividad mental de aprender.

(3) Negociación de los diferentes elementos.

(4) Compromiso recíproco entre el profesor y el alumno de cumplir el contrato.

En general, se trata de una metodología de enseñanza-aprendizaje "personalizada" que permite dirigir el trabajo independiente o no presencial del alumno, promoviendo su autonomía y su capacidad de aprender a aprender.

Los siguientes beneficios para emplear contratos de aprendizaje (Anderson et al., 1996) podrían ser los siguientes:

- Relevancia. Cuando los estudiantes han identificados sus propias necesidades, las actividades se vuelven con toda probabilidad más significativas, relevantes e interesantes para ellos. Los contratos reconcilian las necesidades/intereses de los alumnos con las demandas.

- Autonomía. Los contratos de aprendizaje permiten una considerable libertad para elegir qué aprender y cómo aprenderlo.

- Estructura. Proporciona un esquema formal conocido y compartido por profesores y alumnos para estructurar las actividades de aprendizaje. Al mismo tiempo proporcionan un alto grado de flexibilidad.

- Equidad. Se entiende como diversidad de contenidos y procesos, no como oferta educativa "igual" para todos. Los contratos capacitan a los profesores a responder a 
las necesidades diversas de un amplio rango de estudiantes. Facilitan el acceso a los contextos y la equidad dentro de los cursos.

Los contratos pueden ser utilizados para las siguientes finalidades educativas (García \& Fortea, 2006):

- Promover la autonomía y responsabilidad del alumno.

- Incrementar la motivación e implicación del alumno en su propio aprendizaje (toma de decisiones).

- Estimular la actividad del alumno en el proceso de enseñanza-aprendizaje.

- Atender a las características personales de cada alumno/a: responder a las necesidades, intereses, etc. del alumno como individuo.

- Dirigir académicamente el trabajo independiente del alumno.

- "Democratizar" la educación, promoviendo procesos de negociación y participación del alumno en el proceso de la docencia: desde el establecimiento de los objetivos y contenidos a la evaluación.

- Promover la capacidad de autoevaluación y pensamiento crítico del alumno/a. Especialmente se estimula la capacidad de autorreflexión sobre el propio aprendizaje.

El procedimiento de uso del contrato de aprendizaje podría seguir las siguientes fases (García \& Fortea, 2006), que se pueden observar en la figura 2:

Fase 1: Informar al alumno. A principio del curso el profesor/a presenta los objetivos generales de la asignatura y la planificación docente, con descripción detallada de esta metodología.

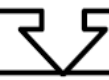

Fase 2: Diagnóstico inicial de la situación de partida del alumno. Facilitar que el alumno analice e identifique su situación de partida en cuanto a aprendizaje, necesidades, intereses, motivaciones, competencias, etc.

Fase 3: Negociación del contrato y orientación del aprendizaje. Entrevista cara a cara profesor/a y alumno/a para acordar los diferentes elementos del contrato. El contrato debe ser individualizado pero a la vez garantizar que cumple con los requisitos/objetivos generales básicos de la asignatura y que sea realista (realizable, claro, etc.). La negociación está presente durante todas las fases. Se pueden elaborar diferentes revisiones del mismo.

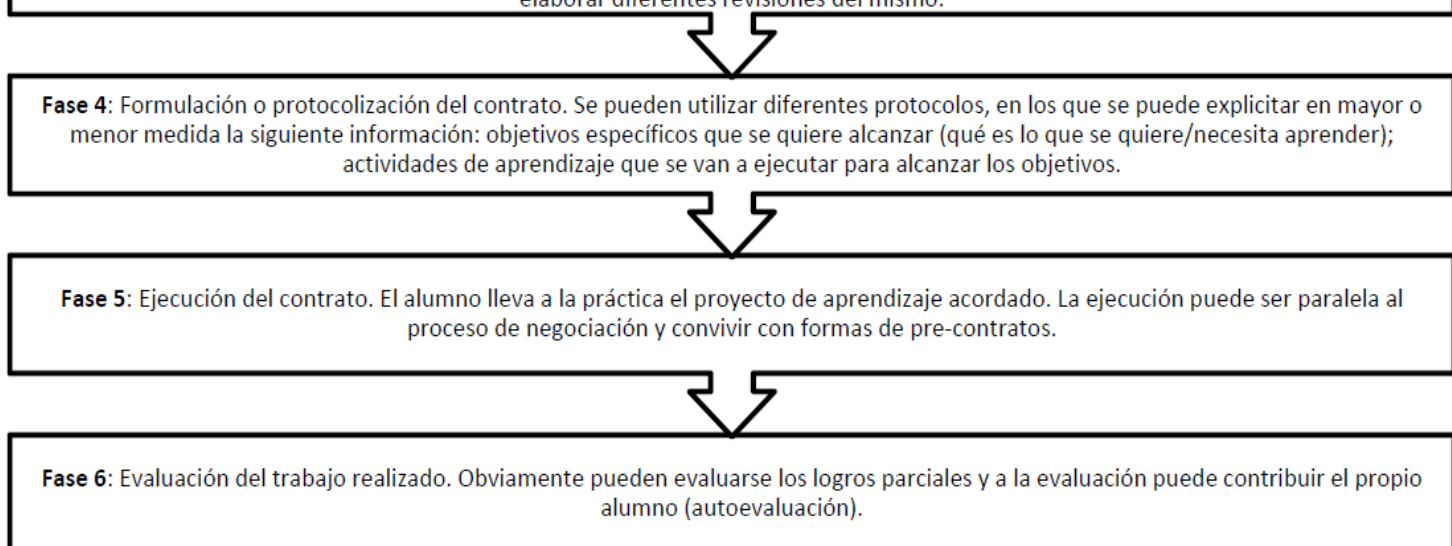

Figura 2. Procedimiento del contrato de aprendizaje.

Fuente: (García \& Fortea, 2006). 
El contrato puede renovarse y cambiarse a lo largo de la vida del mismo bajo el acuerdo del profesor y el alumno.

\section{CONCLUSIONES}

Con el aprendizaje autónomo se potencia la habilidad para descubrir, resolver problemas y tomar decisiones sobre cómo aprender y fomentar el auto-aprendizaje entre los alumnos.

En enseñanza es necesaria la utilización de herramientas que hagan más activo el aprendizaje y que beneficien el auto aprendizaje y la autoevaluación por parte de los estudiantes.

El uso del contrato de aprendizaje implica dotarse de un currículo al servicio de la autonomía y, por tanto, hay que reconsiderar las mismas decisiones que se toman al planificar la propuesta docente (García \& Fortea, 2006).

En el proceso de evaluación del alumno/a, el contrato de aprendizaje implica un seguimiento que posibilita una evaluación continua o de proceso del aprendizaje.

La educación tradicional puede ser complementada por la tecnología, y ésta convertirse en una herramienta pedagógica para favorecer el desarrollo del aprendizaje autónomo.

El trabajo de aprendizaje autónomo permite que los estudiantes se acoplen a su propio ritmo de aprendizaje.

\section{REFERENCIAS BIBLIOGRÁFICAS}

Anderson, G., Bould, D. y Sampson, J. (1996): Learning contracts. A practical guide. London: Kogan Page.

Crispín, M.L. (2011). Aprendizaje autónomo: Orientaciones para la docencia. Universidad iberoamericana. Biblioteca Francisco Xavier Clavigero. México. ISBN: 978-607-417137-2.

García, F. \& Fortea, M. (2006). Ficha metodológica coordinada por Universitat Jaume I. UJI, Mayo 2006.

Martí, E. (2000). "Metacognición y estrategias de aprendizaje”, en Pozo, J.I. y Monereo, C. El aprendizaje estratégico. Madrid: Aula siglo XXI, Santillana.

Martínez Fernández, J.R. (2004). Concepción del aprendizaje, metacognición y cambio conceptual en estudiantes universitarios de Psicología. Tesis doctoral, Universidad de Barcelona.

Monereo, C. (2001). Ser estratégico y autónomo aprendiendo. Unidades didácticas de enseñanza estratégica. Barcelona: GRAO. 
Pérez de Cabrera, L. (2013). El rol del docente en el aprendizaje autónomo: la perspectiva del estudiante y la relación con su rendimiento académico. Rev Diálogos. Enero-Junio de 2013, pp.45-62.

Sanz de Acedo, M.L. (1998). Inteligencia y personalidad en las interfases educativas. Bilbao: Desclée De Brouwer.

Vázquez, Y. A. (2001). Educación basada en competencias. Educar: Revista de educación/nueva época, 16, 1-29. 